\title{
Parton energy loss effect on $Z+$ jet production in high-energy nuclear collisions
}

\author{
Shan-Liang Zhang ${ }^{* a}$, Tan Luo ${ }^{a}$, Xin-Nian Wang ${ }^{a b}$, Ben-Wei Zhang ${ }^{a}$ \\ ${ }^{a}$ Institute of Particle Physics and Key Laboratory of Quarks and Lepton Physics (MOE), Central \\ China Normal University, Wuhan 430079, China \\ ${ }^{b}$ Nuclear Science Division Mailstop 70R0319, Lawrence Berkeley National Laboratory, \\ Berkeley, CA 94740 \\ E-mail: zhangshanlemails.ccnu.edu.cn, luotan@mails.ccnu.edu.cn, \\ xnwang@lbl.gov, bwzhang@mail.ccnu.edu.cn
}

We give a report of medium modification of $\mathrm{Z}+$ jet correlations in $\mathrm{Pb}+\mathrm{Pb}$ collisions at the Large Hadron Collider using Sherpa to generate initial Z+jet at next-to-leading-order matrix element matched to the parton shower, and the Linear Boltzmann Transport Model for jet propagation in the expanding quark-gluon-plasma. Our numerical calculations show excellent agreement with all available observables of $\mathrm{Z}+$ jet simultaneously in both proton + proton and $\mathrm{Pb}+\mathrm{Pb}$ collisions. Our results can well explain the shift of momentum asymmetry $x_{j Z}=p_{T}^{j e t} / p_{T}^{Z}$ as well as its mean values, the suppression of the jet yields per $Z$ trigger $R_{j Z}$ and the modification of azimuthal angle correlations $\Delta \phi_{j z}$. We also demonstrate that it is the energy loss effect on multi-jet from higher order corrections that leads to the suppression of the $\mathrm{Z}+\mathrm{jet}$ correlations in small azimuthal angle difference $\Delta \phi_{j Z}$ region and in small $x_{j Z}$ region. The jet shape reflecting transverse momentum distribution inside the jet is also calculated, which indicates that large fraction of jet energy is carried away from the jet axis in $\mathrm{Pb}+\mathrm{Pb}$ collisions.

International Conference on Hard and Electromagnetic Probes of High-Energy Nuclear Collisions 30 September - 5 October 2018

Aix-Les-Bains, Savoie, France

${ }^{*}$ Speaker. 


\section{Introduction}

Jet production in association with $\mathrm{Z}$ boson provides an ideal probe of the properties of the quark-gluon plasma (QGP) [1]. The outgoing partons interact strongly with the hot and dense medium and lose energy in the QGP [2], while Z bosons will not participate in the strong-interactions directly, escaping the QGP unscathed. Besides, compared to $\gamma+j e t, \mathrm{Z}$ bosons have no fragmentation contributions due to its large mass. Therefore, the $\mathrm{Z}$ boson transverse momentum closely reflects the initial energy of the associated parton that fragments into the final-state jet.

$Z+$ jet correlations quantified as transverse momentum asymmetry $x_{j Z}=p_{T}^{\text {jet }} / p_{T}^{Z}$ as well as its mean value $\left\langle x_{j Z}\right\rangle$, jet yields per $Z$ trigger $R_{j Z}=N_{j Z} / N_{Z}$, and azimuthal correlations $\Delta \phi_{j Z}=$ $\left|\phi_{\text {jet }}-\phi_{Z}\right|$ both in proton+proton $(\mathrm{p}+\mathrm{p})$ and lead+lead $(\mathrm{Pb}+\mathrm{Pb})$ collisions at $5.02 \mathrm{TeV}$ have been measured by the CMS experiment [3]. It is noted that when computing $\Delta \phi_{j Z}$, the next-to-leadingorder (NLO) calculations suffer from divergence in the region $\Delta \phi_{j Z} \sim \pi$, because of soft collinear radiation. Furthermore, even though leading-order (LO) matched parton shower (PS) calculations contain already some higher order corrections from real and virtual contributions, it does not include additional hard radiation from higher order matrix element (ME) calculations, as a consequence of which, it underestimates the azimuthal angle correlations in the small angle difference region $[4,5]$. Motivated by this, we present in this article a state of art calculations of Z+jet production [4], with p+p baseline computed at NLO+PS with Sherpa [6], and the Linear Boltzmann Transport (LBT) model [7] for jet propagation in heavy-ion collisions.

\section{Model setup for $\mathbf{Z}+$ jet in heavy-ion collisions}

Reference $\mathrm{Z}+\mathrm{jet}$ production in $\mathrm{p}+\mathrm{p}$ collisions is simulated using NLO matrix element perturbative calculations matched to the resummation of parton showers $[8,9]$ within the Monte Corlo event generator Sherpa [6] at $\sqrt{s_{N N}}=5.02 \mathrm{TeV}$. NLO +PS calculations of angular correlations and momentum asymmetry for Z+jet agree well with experimental data [3] in all kinematic region in $\mathrm{p}+\mathrm{p}$ collisions [4]. EPPS16 modified npdfs are used to study cold nuclear matter effects, but no modifications are observed as shown in [10].

The Linear Boltzmann Transport (LBT) model is then used to simulate the propagation, energy attenuation of, and medium response induced by jet partons in the QGP [7]. LBT is based on a Boltzmann equation [7]:

$p_{a} \cdot \partial f_{a}\left(p_{a}\right)=-\frac{1}{2} \int \sum_{i=b, c, d} \frac{d^{3} p_{i}}{(2 \pi)^{3} 2 E_{i}} \times\left[f_{a} f_{b}-f_{c} f_{d}\right]\left|M_{a b \rightarrow c d}\right|^{2} \times S_{2}(s, t, u)(2 \pi)^{4} \delta^{4}\left(p_{a}+p_{b}-p_{c}-p_{d}\right)$

where $f_{i}$ are phase-space distributions of partons, $S_{2}(s, t, u)$ is a Lorentz-invariant regulation condition. Elastic scattering is introduced by the complete set of $2 \rightarrow 2$ matrix elements $\left|M_{a b \rightarrow c d}\right|$, and the inelastic scattering is described by the higher-twist formalism for induced gluon radiation $[11,12,13]$.

\section{Numerical results}

To compare with the experimental data, we select the $\mathrm{Z}$ boson and jets according to the kinematic cut adopted by CMS [3]. The information of the evolving bulk matter is provided by $(3+1) \mathrm{D}$ 
hydrodynamics [14]. The underlying event background energy is subtracted event-by-event for $\mathrm{Pb}+\mathrm{Pb}$ collisions following the procedure applied in CMS [15], while no subtraction is applied in $\mathrm{p}+\mathrm{p}$ collisions.

We first fix the only parameter $\alpha_{s}$ that controls the strength of jet-medium interactions via the comparison with the CMS data for Z+jets [3]. When $\alpha_{s}$ is set to 0.2 , our numerical results for the jet yields per $\mathrm{Z}$ trigger $R_{j Z}$ in central $\mathrm{Pb}+\mathrm{Pb}$ collisions show good agreement with CMS data as shown in Fig. 1 (left). $R_{j Z}$ is overall suppressed in $\mathrm{Pb}+\mathrm{Pb}$, because a large fraction of jets lose energy and then their final transverse momenta fall below the threshold $p_{T}^{\text {jet }}=30 \mathrm{GeV}$.

The transverse momentum imbalance between the associated jet and the recoiling $\mathrm{Z}$ boson $x_{j Z}=p_{T}^{j e t} / p_{T}^{Z}$ is presented in Fig. 1 (right). Compared to $\mathrm{p}+\mathrm{p}$ collisions, there is a significant displacements of the peak position of $x_{j Z}$ towards a smaller value in $\mathrm{Pb}+\mathrm{Pb}$, due to jet energy loss in the medium while the transverse momentum of the $\mathrm{Z}$ boson is unattenuated. Multi-jet processes are rather important when $x_{j Z}<0.5$, where the multi-jet energy can hardly exceed half of the energy of the $\mathrm{Z}$ boson in the region $\Delta \phi_{j Z} \geq \frac{7 \pi}{8}$.
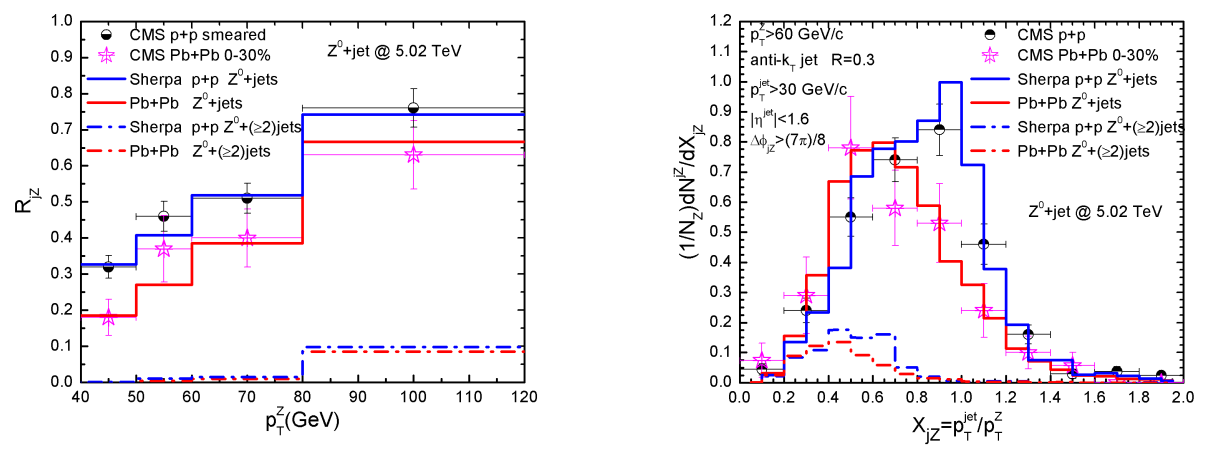

Figure 1: (Color online) Distributions of (Left) $R_{j Z}=N_{j Z} / N_{Z}$ and (Right) $x_{j Z}=p_{T}^{j e t} / p_{T}^{Z}$ in central $\mathrm{Pb}+\mathrm{Pb}$ collisions and $\mathrm{p}+\mathrm{p}$ collisions at $\sqrt{s_{N N}}=5.02 \mathrm{TeV}$.
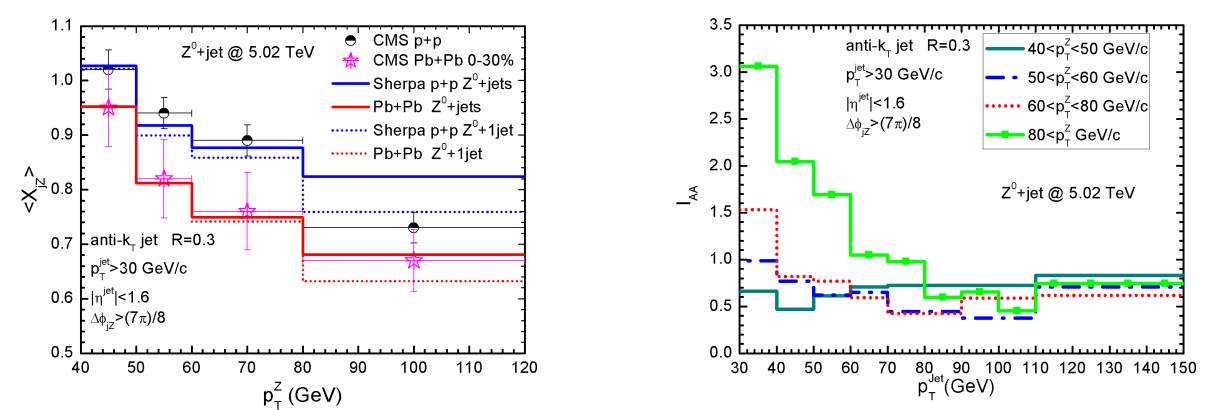

Figure 2: (Color online) (Left) Mean value of momentum imbalance $\left\langle x_{j z}\right\rangle$ of Z+jet in central $\mathrm{Pb}+\mathrm{Pb}$ (red) and $\mathrm{p}+\mathrm{p}$ collisions (blue) at $\sqrt{s_{N N}}=5.02 \mathrm{TeV}$. (Right) $I_{A A}$ as a function of $p_{T}^{\text {jet }}$ in different $p_{T}^{Z}$ bins.

To quantify the relative shift between $\mathrm{p}+\mathrm{p}$ and $0-30 \%$ central $\mathrm{Pb}+\mathrm{Pb}$ collisions, the mean value of the momentum asymmetry $\left\langle x_{j Z}\right\rangle$ is calculated and shown in Fig. 2 (left). It is much smaller in $\mathrm{Pb}+\mathrm{Pb}$ relative to $\mathrm{p}+\mathrm{p}$ collisions. Fig. 2 (right) plots the nuclear modification factor $I_{A A}=\left(d N^{P b+P b} / d p_{T}^{j e t}\right) /\left(d N^{p+p} / d p_{T}^{j e t}\right)$ of the leading jet tagged by a $\mathrm{Z}$ boson. An enhancement is 


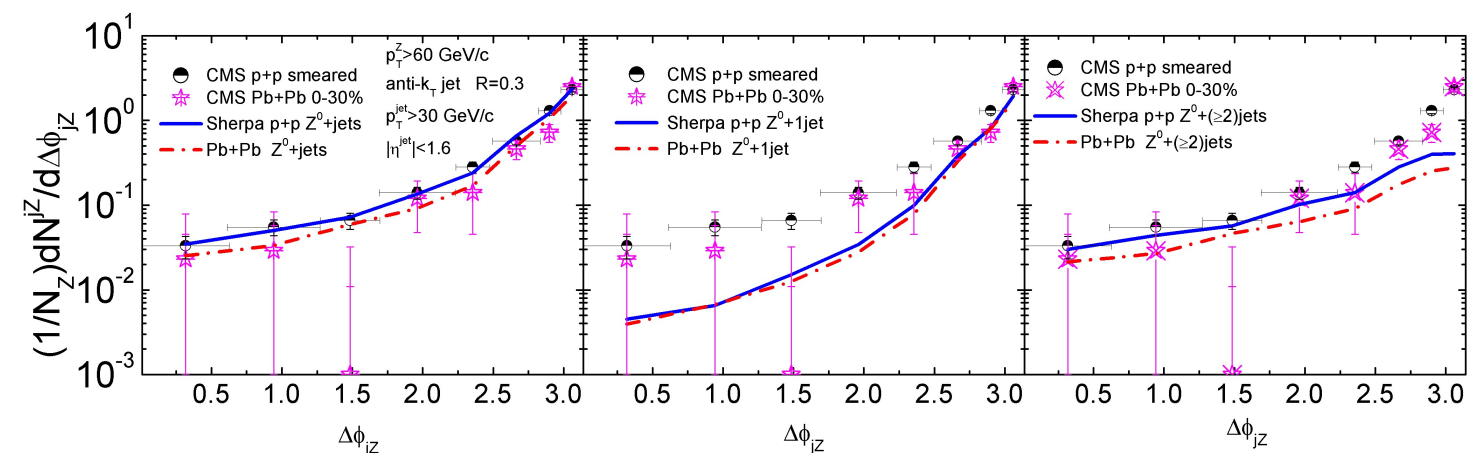

Figure 3: (Color online) Z+jet azimuthal angle correlations $\Delta \phi_{j Z}=\left|\phi_{j e t}-\phi_{Z}\right|$ (left), and the contributions from $\mathrm{Z}$ plus only one jet (middle), and $\mathrm{Z}$ plus more than one jet (right) both in central $\mathrm{Pb}+\mathrm{Pb}$ collisions and $\mathrm{p}+\mathrm{p}$ collisions at $\sqrt{s_{N N}}=5.02 \mathrm{TeV}$.

observed in the $p_{T}^{\text {jet }}<p_{T}^{Z}$ region, and a suppression in $p_{T}^{\text {jet }}>p_{T}^{Z}$ region. We find that $I_{A A}$ is quite sensitive to the kinematic cut due to the steeply falling cross-section in this region .

$\mathrm{Z}+$ jet azimuthal angle correlations $\Delta \phi_{j Z}=\left|\phi_{\text {jet }}-\phi_{Z}\right|$ in $\mathrm{p}+\mathrm{p}$ and $\mathrm{Pb}+\mathrm{Pb}$ are shown in Fig. 3 (left). They are moderately suppressed in $\mathrm{Pb}+\mathrm{Pb}$ collisions. To illustrate the suppression mechanism, separated contributions from $\mathrm{Z}+1$ jet and $\mathrm{Z}$ associated with more than one jet in both $\mathrm{p}+\mathrm{p}$ and $\mathrm{Pb}+\mathrm{Pb}$ collisions are revealed in Fig. 3 . We see that $\mathrm{Z}+1$ jet dominates in the large angle region and there is no significant difference between $\mathrm{p}+\mathrm{p}$ and $\mathrm{Pb}+\mathrm{Pb}$ collisions. The decorrelation of $\Delta \phi_{j Z}$ in the large angle region mainly comes from soft collinear radiation. The transverse momentum broadening of jets due to jet-medium interaction is negligible compared to soft collinear radiation at such high energy scale. The right panel of Fig. 3 illustrates that $\mathrm{Z}+$ multi-jet processes are considerably suppressed in $\mathrm{Pb}+\mathrm{Pb}$ collisions.

In addition to $\mathrm{Z}+\mathrm{jet}$ correlations, we calculated the differential jet profile which describes the radial distribution of transverse momentum inside the jet cone [16]. The differential jet shape in $\mathrm{Pb}+\mathrm{Pb}$ and $\mathrm{p}+\mathrm{p}$ collisions are displayed in Fig. 4. The result is normalized to unity over $r<0.3$. We see that, a large fraction of jet energy is carried in the core of the jet within $r<0.1$. To quantify the modification, we present the ratio of the jet shape in $\mathrm{Pb}+\mathrm{Pb}$ to that in pp collisions in Fig. 4 (right). We observe a depletion in the region $0.05<r<0.1$ and an enhancement at large radius $r>0.1$. It indicates that the energy is redistributed in $\mathrm{Pb}+\mathrm{Pb}$ collisions due to jet-medium interactions and large amount of jet energy is carried by particles far away from the jet axis.

This work has been supported by NSFC of China with Project Nos. 11435004, and NSF under grant No. ACI-1550228 and U.S. DOE under Contract No. DE-AC02-05CH11231.

\section{References}

[1] R. B. Neufeld, I. Vitev and B.-W. Zhang, The Physics of $Z^{0} / \gamma^{*}$-tagged jets at the LHC, Phys. Rev. C 83, 034902 (2011)

[2] M. Gyulassy, I. Vitev, X. N. Wang and B. W. Zhang, Jet quenching and radiative energy loss in dense nuclear matter, In *Hwa, R.C. (ed.) et al.: Quark gluon plasma* 123-191 [nucl-th/0302077].

[3] A. M. Sirunyan et al. [CMS Collaboration], Study of Jet Quenching with Z+ jet Correlations in $\mathrm{Pb}-\mathrm{Pb}$ and pp Collisions at $\sqrt{s}_{N N}=5.02 \mathrm{TeV}$, Phys. Rev. Lett. 119, no. 8, 082301 (2017) 

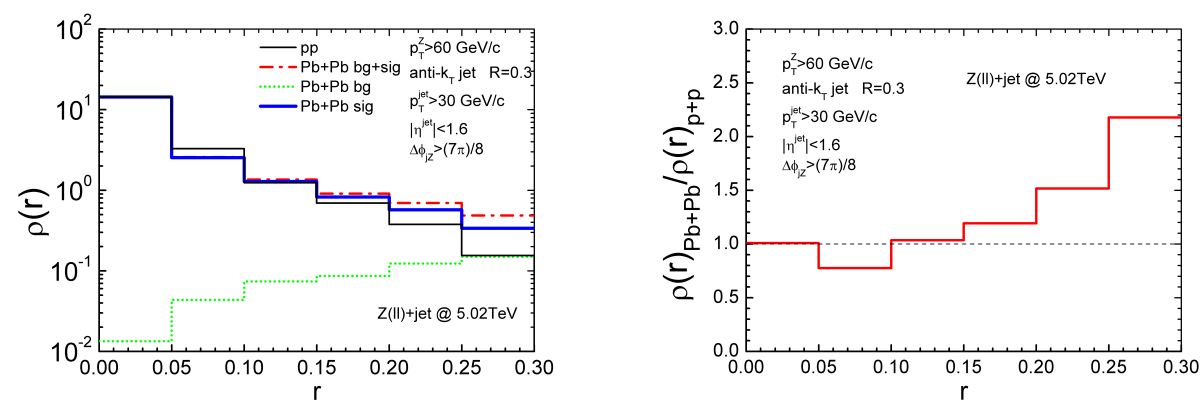

Figure 4: (Color online) Differential jet shape $\rho(r)$ of jets triggered by $\mathrm{Z}$ boson in $0-30 \%$ central $\mathrm{Pb}+\mathrm{Pb}$ and $\mathrm{p}+\mathrm{p}$ collisions at $\sqrt{s}_{N N}=5.02 \mathrm{TeV}$ as well as the ratio of jet shape in central $\mathrm{Pb}+\mathrm{Pb}$ to that in $\mathrm{p}+\mathrm{p}$ collisions.

[4] S. L. Zhang, T. Luo, X. N. Wang and B. W. Zhang, Z+jet correlation with NLO-matched parton-shower and jet-medium interaction in high-energy nuclear collisions, Phys. Rev. C 98, 021901 (2018)

[5] W. Dai, S. Wang, S. L. Zhang, B. W. Zhang and E. Wang, Transverse Momentum Balance and Angular Distribution of $b \bar{b}$ Dijets in Pb+Pb collisions, arXiv:1806.06332 [nucl-th].

[6] T. Gleisberg, S. Hoeche, F. Krauss, M. Schonherr, S. Schumann, F. Siegert and J. Winter, Event generation with SHERPA 1.1, JHEP 0902, 007 (2009)

[7] Y. He, T. Luo, X. N. Wang and Y. Zhu, Linear Boltzmann Transport for Jet Propagation in the Quark-Gluon Plasma: Elastic Processes and Medium Recoil, Phys. Rev. C 91, 054908 (2015)

[8] S. Hoche, F. Krauss, M. Schonherr and F. Siegert, NLO matrix elements and truncated showers, JHEP 1108, 123 (2011)

[9] S. Hoeche, F. Krauss, M. Schonherr and F. Siegert, QCD matrix elements + parton showers: The NLO case, JHEP 1304, 027 (2013)

[10] P. Ru, S. A. Kulagin, R. Petti and B. W. Zhang, Study of $W^{ \pm}$and Z boson production in proton-lead collisions at the LHC with Kulagin-Petti nuclear parton distributions, Phys. Rev. D 94, no. 11, 113013 (2016).

[11] X. F. Guo and X. N. Wang, Multiple scattering, parton energy loss and modified fragmentation functions in deeply inelastic e A scattering, Phys. Rev. Lett. 85, 3591 (2000)

[12] B. W. Zhang, E. Wang and X. N. Wang, Heavy quark energy loss in nuclear medium, Phys. Rev. Lett. 93, 072301 (2004) [nucl-th/0309040].

[13] A. Schafer, X. N. Wang and B. W. Zhang, Multiple Parton Scattering in Nuclei: Quark-quark Scattering, Nucl. Phys. A 793, 128 (2007)

[14] L. Pang, Q. Wang and X. N. Wang, Effects of initial flow velocity fluctuation in event-by-event $(3+1) D$ hydrodynamics, Phys. Rev. C 86, 024911 (2012)

[15] O. Kodolova, I. Vardanian, A. Nikitenko and A. Oulianov, The performance of the jet identification and reconstruction in heavy ions collisions with CMS detector, Eur. Phys. J. C 50, 117 (2007).

[16] I. Vitev, S. Wicks and B. W. Zhang, A Theory of jet shapes and cross sections: From hadrons to nuclei, JHEP 0811, 093 (2008) 